no 19 (primavera de 2021)

Varia

\title{
Aprendizaje de francés a través de videojuegos cooperativos: Portal 2
}

\author{
Juan Manuel MUÑOZ GoNZÁLEZ \\ Universidad de Córdoba \\ m02mugoj@uco.es \\ https://orcid.org/0000-0001-9332-0465
}

\author{
Carmen de CASTro CASTRo \\ Universidad de Córdoba \\ ff1castr@uco.es \\ https://orcid.org/0000-0002-4562-0196
}

\section{Ana Isabel BrAzo-MiLLÁN \\ Universidad de Córdoba}

abmillan@uco.es

https://orcid.org/0000-0002-5775-0094

\section{Resumen}

Partimos de una experiencia didáctica centrada en el uso del videojuego cooperativo Portal 2 y hemos descrito la experiencia de nuestro alumnado en cuanto al aprendizaje de FLE a nivel universitario mediante un cuestionario de elaboración propia. En él se han analizado las diferencias estadísticas significativas siguiendo un método descriptivo y cuantitativo. Los resultados consideran las cuatro dimensiones obtenidas en el análisis factorial exploratorio y se presentan las relaciones entre los cuatro factores, así como el establecimiento de un modelo predictor de la valoración de la experiencia. Las conclusiones revelan que la incorporación de un videojuego cooperativo y la inclusión de sesiones didácticas ha llevado a una consideración bastante positiva del alumnado con respecto a la dinámica de aprendizaje planteada.

Palabras clave: enseñanza de idiomas, lengua extranjera, gamificación, universidad, nuevas tecnologías.

\section{Résumé}

Nous partons d'une expérience didactique centrée sur l'usage du jeu-vidéo coopératif Portal 2, à partir de laquelle nous avons décrit l'expérience de nos étudiants quant à l'apprentissage de FLE dans l'université moyennant un questionnaire d'élaboration propre. La méthode suivie a été descriptive et quantitative. Les résultats considèrent les 4 dimensions tirées de l'analyse factorielle exploratoire, et dont les résultats descriptifs ont été présentés, de même que l'existence de différences statistiquement significatives selon les variables indépendantes de cette étude. Un modèle prédicteur de l'évaluation de l'expérience a été établi. Les conclusions nous permettent d'affirmer que l'incorporation d'un jeu-vidéo coopératif et l'inclusion de

* Artículo recibido el 28/05/2020, aceptado el 8/01/2021. 
sessions didactiques nous ont menés à une considération assez positive de la part des étudiants face à la dynamique proposée.

Mots clé : enseignement d'idiomes, langue étrangère, gamification, université, nouvelles technologies.

\begin{abstract}
We describe the didactic experience of our students in learning the French language using the cooperative video game Portal 2 through a questionnaire created by the authors. The method has been descriptive and quantitative. The results consider the 4 dimensions obtained in the exploratory factor analysis, while presenting the descriptive results of each dimensions and the existence of statistically significant differences according to the study's independent variables. The relationship between the 4 factors are presented, as well as the establishment of a predictive model of experience evaluation. Our findings lead us to conclude that the incorporation of a cooperative video game and the inclusion of didactic sessions are linked to a positive attitude of the students towards the dynamics proposed.
\end{abstract}

Keywords: language training, second language, gamification, university, new technologies.

\title{
1. Introducción
}

En la actualidad, ya no podemos hablar de novedad cuando planteamos el uso del videojuego en la enseñanza de una lengua extranjera (De Haan, 2011; Higgins, Xiao y Katsipataki, 2012). Numerosos estudios se han realizado a nivel internacional $y$, aunque algunos de los resultados muestran que ni es fácil su implantación en el marco de la enseñanza, ni la motivación o el aprendizaje de la Lengua Extranjera (LE) mejora exponencialmente con su uso (Brazo-Millán, Muñoz González y Castro de Castro, 2018; Klimova y Kacet, 2017), sí es cierto que la gamificación ya forma parte de los actuales modelos de enseñanza y que incluir los videojuegos como herramienta educativa en LE es un objetivo evidente (Brazo-Millán, 2020).

Desde la perspectiva del aprendizaje de una LE, a la hora de elegir un videojuego serio o uno comercial para su uso didáctico, la calidad de los primeros no puede equipararse a la de los conocidos productos, que cuentan con una financiación y capital humano difícilmente igualables y que gozan ya de una inmersión real en el mercado (Dickey, 2011; Kinzie y Joseph, 2008). De estos últimos, el atractivo estético, la jugabilidad y el desarrollo de la trama tienen detrás grandes equipos y enormes presupuestos. En cualquier caso, estamos de acuerdo con Rosas et al. (2003) en que, para fines de aprendizaje de idiomas, es importante seleccionar un videojuego comercial donde el lenguaje desempeñe un papel primordial en el logro del objetivo final del juego, es decir, en el éxito; de modo que, mientras los alumnos-jugadores disfrutan jugando, también estarían involucrados en el procesamiento del lenguaje. Teniendo en cuenta que la diferencia principal entre videojuegos educativos (serios) y videojuegos comerciales radica en que mientras los primeros prestan una atención estricta al contenido, 
los comerciales se centran en elementos estéticos, el juego ideal sería aquel que integrara ambas características, para crear un resultado atractivo tanto en términos de contenido como de apariencia (Ebrahimzadeh y Alavi, 2017).

A este respecto, debemos considerar que los videojuegos comerciales, en su objetivo inicial, están diseñados para ser utilizados de forma lúdica, fuera de todo contexto escolar, y entendemos que su didactización, por lo tanto, supone un uso secundario de los mismos (Brazo-Millán et al., 2018). No obstante, a diferencia de otros materiales menos elaborados, es difícil sustraerse a su cohesión y, sobre todo, al aliciente de enfrentarse a un reto y una recompensa: ganar (Casañ-Pitarch, 2017). Jugar a este tipo de videojuegos puede tener un efecto positivo en la capacidad de comunicación, la adaptabilidad y el ingenio de los estudiantes adultos, convirtiéndose en una herramienta adecuada en la educación superior, en general, y en la LE, en particular (Barr, 2017; Dayoub, 2019).

\subsection{El juego cooperativo}

A pesar de que la mayoría de los videojuegos suelen utilizarse de manera individual, también muchas empresas proponen modos de juego colectivo, ya sea del tipo «multijugador» o "cooperativo». Dada nuestras anteriores experiencias donde los aspectos más negativos provenían de dificultades técnicas como el desconocimiento de los videojuegos en general, que hacía que buena parte de nuestro alumnado se sintiera perdido y dedicara mucho tiempo a resolver dudas (Brazo-Millán et al., 2018; De Castro Castro, Muñoz González y Brazo-Millán, 2018), en nuestra nueva propuesta hemos elegido un reconocido juego cooperativo: Portal 2, suponiendo que la colaboración entre los jugadores mejoraría la capacidad de resolver los puzles, avanzar en el juego y obtener así una recompensa tanto individual como en pareja. Tal y como Thorne, Black y Sykes (2009) sugieren implícitamente, el juego cooperativo crearía una comunidad de práctica en la que la cooperación ampliaría el conocimiento y la comunicación social, beneficiando, como describen Creighton y Szymokowic (2014), la interacción social de los alumnos dentro del aula.

Los videojuegos cooperativos de lógica (también conocidos como «videojuegos de puzle») implican una verdadera cooperación para ser resueltos. No hay enfrentamiento, ni competencia, ni lucha alguna entre los avatares y es necesaria la colaboración de ambos jugadores para avanzar en el juego. Cuando el juego propone dos personajes y dos pantallas de juego, como es el caso de Portal 2, ambos miembros de la pareja de jugadores desarrollan las mismas competencias, lo que crea una gran conexión entre los dos integrantes del grupo y desaparece la desigualdad en la responsabilidad en el trabajo, como ocurre en grupos cooperativos de trabajo «online» (Chiong y Jovanovic, 2012).

El videojuego Portal 2 trata de un juego de lógica en primera persona desarrollado por Valve Corporation. En su modo cooperativo, dos robots deben coordinarse 
para resolver distintos escenarios con pruebas. El objetivo es pasar por cinco tipos de pruebas con el uso de diferentes elementos.

\subsection{La comunicación oral en francés en el juego cooperativo}

Desde la publicación y, más tarde, la aplicación del Marco Común Europeo de Referencia para las Lenguas (MCER) a la enseñanza de Francés Lengua Extranjera (FLE), el trabajo en clase se ha visto integrado en la perspectiva accional: tareas y proyectos aparecen en la actualidad de manera frecuente dentro de las planificaciones docentes y, en todos los niveles de enseńanza, los grupos de trabajo o el trabajo por tándem es algo usual. ¿Dónde cabría, pues, el uso del videojuego?

El videojuego puede llegar a ser una alternativa al mundo real cuando el profesor no tiene la posibilidad de proponer tareas propias de la vida real, destacando el carácter activo del estudiante en un aprendizaje constructivista, pues el jugador es puesto en situación, sumergido en el universo virtual, y llevado a estructurar su saber mediante la observación, la acción y la experimentación, aprendiendo por la acción y, sobre todo, por imitación, y actuando por intento/error (Ollivier, 2014; Schmoll, 2017). Tanto el juego como la tarea propuesta en el MCER persiguen una meta, un resultado: ganar, por medio de estrategias. El aspecto interactivo de este tipo de videojuegos convierte al alumno o alumna en un actor real de la historia al poder influir en su desarrollo (Kessler, 1992).

\section{Metodología de la innovación educativa en FLE 2.1. Contexto de juego}

El contexto didáctico, por su parte, proporciona una colectividad de jugadores de un rango de edad y similares experiencias de vida, lo que constituye un buen punto de partida para crear una situación de juego cooperativo. Para ayudar a la inmersión del alumnado en el contexto TIC, son bienvenidos alumnos colaboradores que tengan experiencia como jugadores y echen una mano a los menos iniciados. A diferencia de otras propuestas donde se limitan a la utilización de escenas sueltas del videojuego mediante equipos formados por lectores, jugadores y observadores (Ebrahimzadeh y Alavi, 2017), el uso del videojuego que nosotros planteamos mantiene las principales características de motivación, reto y recompensa.

Partiendo de nuestra experiencia previa con videojuegos serios para la enseñanza del francés, con el videojuego Les Eonautes (De Castro Castro et al., 2018) constatamos que, en la opinión del alumnado, a pesar de valorar su excelente construcción metodológica, no llegaba a ser considerado como un verdadero videojuego ni a atraer a los estudiantes. En cuanto a proponer un contexto de juego fuera del aula en la que los estudiantes trabajaran de forma individual (Brazo-Millán et al., 2018), videojuegos como Scribblenauts y Broken Sword les plantearon inseguridades y dificultades técnicas que solo los estudiantes con perfil de jugadores eran capaces de resolver. Por ello, tras sus comentarios, recogidos en los artículos citados, hemos planteado una sesión de 
juego didáctica en donde se prime la cooperación y se auxilie a los menos instruidos en tecnología y videojuegos.

\subsection{Descripción de la experiencia}

Planteamos la experiencia en tres fases:

1a Fase: Práctica oral convencional.

Hemos creído conveniente realizar una primera práctica oral convencional por parejas para favorecer la confianza entre ellos y, así, agilizar, en lo posible, el discurso oral en FLE. Esta primera práctica ha consistido en realizar una tarea de ordenación lingüística de textos que entrañaba la expresión de opiniones, de preguntas y órdenes. Dicha práctica fue grabada por ellos en un audio de unos 15 minutos de duración.

$2^{\text {a }}$ Fase: Práctica de instalación del videojuego, instrucciones de uso y visionado de ejemplos de jugadores.

Después de nuestras anteriores experiencias (Brazo-Millán et al., 2018; De Castro Castro et al., 2018) y con el objeto de subsanar en lo posible los problemas técnicos que se habían creado, realizamos una sesión en la que los alumnos instalaron el videojuego en su portátil mediante un tutorial y accedieron a ejemplos de uso en francés, así como a un vocabulario específico, también en francés, de manejo del teclado, ratón, pantalla, etc.

$3^{a}$ Fase: Sesión de juego.

Se han requerido 2 espacios dotados de equipamiento informático (Pc, auriculares con micrófono, conexión a internet y aplicación de grabación de audio) donde los estudiantes, organizados por parejas, formaron dos grupos de alumnos. Cada miembrojugador pertenecía a un grupo. Cada jugador se instaló en un espacio diferente, es decir, que cada miembro de la pareja jugó desde un aula diferente a la de su compañero/a de juego. De esta manera, tenían que comunicarse mediante la plataforma Steam para poder jugar, siendo obligatoria la utilización del idioma francés desde el inicio hasta el final de la experiencia.

La propia plataforma Steam pone a disposición de los usuarios una aplicación de chat y comunicación mediante voz síncrona que permite la comunicación entre los jugadores en el desarrollo del juego cooperativo. Por lo tanto, la plataforma facilita la creación de comunidades de jugadores para el intercambio de experiencias relacionadas con la dinámica de aprendizaje vivida. De esta manera, todo queda centralizado, incluyendo la adquisición de los títulos como el Portal 2, empleado para esta innovación.

Tras la partida de juego (sesión con una duración de 4 horas), cada alumnojugador rellenó el cuestionario, que habíamos elaborado con el fin de extraer a posteriori los resultados de la innovación, así como unas preguntas abiertas que nos sirvieran para que los estudiantes dejaran constancia de sus reflexiones durante el desarrollo de la sesión. 
Asimismo, durante la partida, los alumnos-jugadores también se grabaron en audio -de forma individual- durante 20 minutos. Estos audios nos fueron enviados por parejas de juego tras la partida a los docentes-investigadores.

Aunque los docentes-investigadores hemos sido los encargados de llevar a cabo la dinámica de aprendizaje, así como la recogida de datos en ambas titulaciones, en las fases 2 y 3 hemos contado con la asistencia de alumnos colaboradores que conocían, no solo los aspectos técnicos de instalación y manejo del videojuego, sino también el desarrollo de Portal 2, con lo que pudieron ayudar a aquellos alumnos con alguna dificultad. Ellos también se comunicaban esencialmente en francés con los estudiantes participantes en esta innovación.

El principal objetivo de esta innovación educativa consiste en describir la experiencia del alumnado de los Grados de Maestro de Educación Primaria ( $3^{\text {er }}$ y $4^{\mathrm{o}}$ cursos) y de Traducción e Interpretación ( $2^{\circ}$ curso) de la Universidad de Córdoba (España) en cuanto a la enseńanza-aprendizaje del francés como LE mediante el uso de videojuegos cooperativos. Este objetivo general se puede dividir en varios objetivos específicos, que se concretan a continuación:

1. Describir las opiniones del alumnado de los Grados de Maestro de Educación Primaria y de Traducción e Interpretación respecto a la experiencia vivida en una dinámica de aprendizaje del idioma francés basada en videojuegos cooperativos.

2. Analizar la existencia de diferencias estadísticamente significativas en relación con el sexo, la edad, la titulación y el curso del alumnado, así como el uso de dispositivos electrónicos.

3. Comprobar las relaciones existentes entre las diferentes dimensiones del cuestionario.

4. Establecer un modelo para predecir la valoración del videojuego Portal 2 en la enseñanza-aprendizaje del francés en función del perfil jugador de los estudiantes, así como de la calidad del título.

\subsection{Método}

El método utilizado en esta innovación es descriptivo y cuantitativo debido al carácter de los datos recogidos. Los análisis llevados a cabo y mediante los cuales se pretende dar respuesta a los objetivos planteados han sido de tipo descriptivo (frecuencias, porcentajes, medidas de tendencia central y de dispersión), inferenciales con significación estadística, correlación y regresión. A partir de estos, se han interpretado los resultados y establecido las conclusiones.

\subsection{Muestra y participantes}

El tipo de muestreo llevado a cabo en el estudio corresponde con el procedimiento de muestreo no probabilístico o de conveniencia (Otzen y Manterola, 2017), 
ya que la dinámica de aprendizaje diseñada solo se pudo realizar en los grupos de sujetos a los que los investigadores de este estudio impartían clase durante el curso académico 2018-2019.

La muestra estuvo formada por 108 estudiantes: 56 correspondientes al Grado de Maestro de Educación Primaria (51,9\%) y 52 alumnos pertenecientes al Grado de Traducción e Interpretación $(48,1 \%)$. El perfil del alumnado en cuanto a la edad y el sexo se puede observar en la siguiente tabla:

\begin{tabular}{|l|c|c|c|}
\hline \multicolumn{1}{|c|}{ Edad } & Mujeres & Hombres & Total \\
\hline 18-20 años & $49.1 \%$ & 7.4 & $56.5 \%$ \\
\hline 21-23 años & $27.8 \%$ & $5.6 \%$ & $33.3 \%$ \\
\hline 24-26 ańos & $2.8 \%$ & $1.9 \%$ & $4.6 \%$ \\
\hline más de 26 años & $5.6 \%$ & $0 \%$ & $5.6 \%$ \\
\hline
\end{tabular}

Tabla 1. Perfil del alumnado participante

El nivel de competencia comunicativa en francés de acuerdo con el MCER recomendado a nuestros alumnos para cursar dichas asignaturas es de un B1.

\subsection{Instrumento}

El instrumento de recogida de datos utilizado para llevar a cabo este estudio ha consistido en un cuestionario elaborado ah hoc, que hemos denominado Cuestionario sobre la enseñanza-aprendizaje del francés a través del videojuego Portal 2, compuesto de 35 ítems. La validez de este ha sido medida a través de un análisis factorial exploratorio (AFE), utilizando para la ejecución de la prueba el método de Implementación óptima del Análisis Paralelo (PA) (Timmerman y Lorenzo-Seva, 2011), junto con un proceso de extracción de factores comunes "mínimos cuadrados no ponderados robusto» (RULS), considerando un procedimiento de rotación «Oblimin Ponderado» (LorenzoSeva, 2000). Los diferentes índices obtenidos fueron los siguientes: Kaiser-Meyer-Olkin $(\mathrm{KMO})$ con valor de .90; test de esfericidad de Barllett con $\mathrm{p}=0.000$; análisis de residuales con $\mathrm{RMSR}=0,045$ y varianza total explicada $61,55 \%$, considerados adecuados para la validación del modelo. La estructura factorial obtenida muestra la existencia de 4 factores, que se denominan a continuación: 1. Valoración de la experiencia, 2. Aportaciones del videojuego en la enseñanza-aprendizaje del francés, 3. Calidad de los sonidos/subtítulos, y 4. Recursos de apoyo usados durante la dinámica de aprendizaje (ver tabla 2). 


\begin{tabular}{|c|c|c|c|c|}
\hline & $\begin{array}{l}\text { Factor } 1 . \text { Valora- } \\
\text { ción de la } \\
\text { experiencia }\end{array}$ & $\begin{array}{l}\text { Factor 2. Aporta- } \\
\text { ciones del video- } \\
\text { juego en el apren- } \\
\text { dizaje del francés }\end{array}$ & $\begin{array}{l}\text { Factor 3. Calidad } \\
\text { de los sonidos/sub- } \\
\text { títulos }\end{array}$ & $\begin{array}{l}\text { Factor 4. Recursos } \\
\text { de apoyo usados } \\
\text { durante la diná- } \\
\text { mica de aprendizaje }\end{array}$ \\
\hline Item 1 & .832 & & & \\
\hline Item 2 & .644 & & & \\
\hline Item3 & 1.016 & & & \\
\hline Item 4 & .945 & & & \\
\hline Item 5 & .767 & & & \\
\hline Item6 & .448 & & & \\
\hline Item 7 & .560 & & & \\
\hline Item 8 & .766 & & & \\
\hline Item9 & .370 & & & \\
\hline Item 10 & .808 & & & \\
\hline Item 11 & & .569 & & \\
\hline Item 12 & & .801 & & \\
\hline Item 13 & & .845 & & \\
\hline Item 14 & & .430 & & \\
\hline Item 15 & & .861 & & \\
\hline Item 16 & & .795 & & \\
\hline Item 17 & & .494 & & \\
\hline Item 18 & & .686 & & \\
\hline Item 19 & & .472 & & \\
\hline Item 20 & & .563 & & \\
\hline Item 21 & & .510 & & \\
\hline Item 22 & & & .587 & \\
\hline Item23 & & & .306 & \\
\hline Item 24 & & & .686 & \\
\hline Item 25 & & & .369 & \\
\hline \multicolumn{5}{|l|}{ Item 26} \\
\hline Item27 & & & & .531 \\
\hline Item 28 & & & & .318 \\
\hline Item 29 & & & & .739 \\
\hline Item30 & & & & .802 \\
\hline
\end{tabular}

Tabla 2. Estructura de 4 factores obtenida en el AFE

Por último, se comprobó la fiabilidad del instrumento a través del enfoque de consistencia interna (Merino-Soto, 2016), consiguiendo un valor en el Alfa de Cronbach de 0.92, en el caso del instrumento completo, así como de 0.89 en el factor 1; 0.92 en el factor 2; 0.76 en el factor 3 y 0.63 en el factor 4, lo cual refleja una fiabilidad alta del instrumento, así como de los factores 1 y 2 ; y una fiabilidad aceptable en el caso de los fatores 3 y 4 .

\subsection{Procedimiento}

El cuestionario fue administrado a los sujetos de la experiencia de innovación vía «online» al finalizar la dinámica de enseñanza-aprendizaje del francés como LE 
basada en el uso del videojuego Portal 2, dedicando 30 minutos para su cumplimentación. Durante su aplicación, los investigadores del proyecto estuvieron presentes para resolver posibles dudas de comprensión que pudieran surgir.

\subsection{Análisis de datos}

Los análisis realizados en este estudio, a través de los cuales se ha perseguido dar respuesta a los diferentes objetivos planteados, son los siguientes:

- Análisis descriptivo de las 4 dimensiones del cuestionario, calculando la distribución de frecuencias, los estadísticos de medida central y las medidas de dispersión.

- Análisis de varianza para comprobar si existía relación entre las dimensiones del cuestionario y las variables independientes: sexo, edad, titulación, curso y dispositivo utilizado para jugar a videojuegos. Para ello, se emplearon las pruebas t-Student y ANOVA de un factor, utilizando el software SPSS 23.

- Estudio de las relaciones entre las diferentes dimensiones del cuestionario a través de la correlación de Pearson.

- Regresiones lineales múltiples para predecir la valoración de la experiencia vivida en función de las aportaciones del videojuego en la enseñanza-aprendizaje del francés, la calidad del sonido y los subtítulos, así como los recursos de apoyo usados durante la dinámica de aprendizaje.

\section{Resultados}

En primer lugar, se muestran los resultados considerando las 4 dimensiones obtenidas en el análisis factorial exploratorio. Al respecto, se exponen los resultados descriptivos de cada una de las dimensiones, así como la existencia de diferencias estadísticamente significativas, atendiendo a las variables independientes del estudio (sexo, edad, titulación, curso y dispositivo utilizado para jugar). Finalmente, se presentan las relaciones entre los 4 factores, así como el establecimiento de un modelo predictor de la valoración de la experiencia en función de las aportaciones del videojuego en la enseñanza-aprendizaje del francés, la calidad de los sonidos/subtítulos de este, así como los recursos de apoyo usados durante la dinámica de aprendizaje (ver tabla 3):

\begin{tabular}{|l|l|c|c|}
\hline \multicolumn{1}{|c|}{ Factores } & \multicolumn{1}{|c|}{ Ítem } & \multicolumn{1}{c|}{ M } & \multicolumn{1}{c|}{ DT } \\
\hline & 1. El juego mantiene toda mi atención & 3.75 & 1.04 \\
\hline & 2. El juego es divertido & 3.63 & .93 \\
\hline $\begin{array}{l}\text { 3. Hay que pensar de forma estratégica para superar } \\
\text { Factor 1: Valoración de } \\
\text { la experiencia }\end{array}$ & 4.56 & .67 \\
\hline & $\begin{array}{l}\text { 4. Es un juego que exige cooperación con tu compa- } \\
\text { nero/a }\end{array}$ & 4.79 & .44 \\
\hline & $\begin{array}{l}\text { 5. Ha sido divertido jugar en modo colaborativo } \\
\text { 6. Pienso que se debe fomentar esta forma de juego } \\
\text { para el aprendizaje de idiomas }\end{array}$ & 4.18 & .95 \\
\hline
\end{tabular}




\begin{tabular}{|c|c|c|c|}
\hline & $\begin{array}{l}\text { 7. La metodología planteada en clase me ha resultado } \\
\text { novedosa }\end{array}$ & 4.06 & .91 \\
\hline & $\begin{array}{l}\text { 8. A pesar de las dificultades vividas ha sido una } \\
\text { buena experiencia }\end{array}$ & 3.95 & .97 \\
\hline & $\begin{array}{l}\text { 9. La práctica del juego en español ha resultado útil } \\
\text { para el desarrollo de la dinámica de aprendizaje en } \\
\text { francés }\end{array}$ & 3.38 & 1.16 \\
\hline & $\begin{array}{l}\text { 10. He visto mi progresión en el juego durante la se- } \\
\text { sión }\end{array}$ & 3.94 & 1.05 \\
\hline & $\begin{array}{l}\text { 11. Jugar en una plataforma en francés (Steam) me } \\
\text { aporta conocimientos tecnológicos en dicha lengua }{ }^{1}\end{array}$ & 3.63 & 1.01 \\
\hline & $\begin{array}{l}\text { 12. Considero útil este conocimiento en mi forma- } \\
\text { ción }\end{array}$ & 3.30 & 1.17 \\
\hline & 13. El juego mejora mi comprensión oral & 3.67 & 1.15 \\
\hline & 14. El juego mejora mi comprensión escrita & 2.56 & 1.03 \\
\hline Factor 2. Aportaciones & 15. El juego mejora mi expresión oral & 3.82 & 1.09 \\
\hline del videojuego en la en- & 16. El juego ha ampliado mi léxico en francés & 3.39 & 1.04 \\
\hline $\begin{array}{l}\text { señanza-aprendizaje del } \\
\text { francés }\end{array}$ & $\begin{array}{l}\text { 17. He practicado la expresión oral en francés para } \\
\text { jugar }\end{array}$ & 4.00 & 1.05 \\
\hline & $\begin{array}{l}\text { 18. Creo que en la dinámica de aprendizaje se da un } \\
\text { uso real de la lengua para realizar tareas concretas }\end{array}$ & 3.64 & 1.04 \\
\hline & $\begin{array}{l}\text { 19. Las indicaciones proporcionadas para jugar esta- } \\
\text { ban claras }\end{array}$ & 3.21 & 1.08 \\
\hline & 20. Ha resultado útil la experiencia a nivel individual & 3.50 & 1.00 \\
\hline & 21. Ha resultado útil la experiencia a nivel de grupo & 3.86 & .95 \\
\hline & $\begin{array}{l}\text { 22. Las orientaciones de Wheatley (sistema) se com- } \\
\text { prenden bien }\end{array}$ & 3.14 & 1.12 \\
\hline $\begin{array}{l}\text { Factor 3. Calidad de so- } \\
\text { nidos/subtítulos }\end{array}$ & $\begin{array}{l}\text { 23. La ambientación sonora refuerza los aspectos vi- } \\
\text { suales }\end{array}$ & 3.41 & 1.00 \\
\hline & 24. Los subtítulos son legibles & 3.63 & 1.10 \\
\hline & 25. Los subtítulos me han ayudado a jugar & 3.29 & 1.24 \\
\hline & 26. He revisado el tutorial antes de la sesión de juego & 2.88 & 1.58 \\
\hline & 27. He preguntado a amigos/as sobre cómo jugar & 3.34 & 1.49 \\
\hline $\begin{array}{l}\text { Factor } 4 \text {. Kecursos de } \\
\text { apoyo usados durante la }\end{array}$ & 28. He utilizado el diccionario como ayuda & 2.34 & 1.39 \\
\hline dinámica de aprendizaje & 29. He utilizado el traductor como ayuda & 2.34 & 1.39 \\
\hline & $\begin{array}{l}\text { 30. He preguntado al profesor/colaborador para re- } \\
\text { cibir ayuda }\end{array}$ & 4.16 & 1.06 \\
\hline
\end{tabular}

Tabla 3. Distribución de frecuencias de los ítems del Cuestionario sobre la enseñanza-aprendizaje del francés a través del videojuego Portal 2

\footnotetext{
${ }^{1}$ Se trata del vocabulario específico del manejo del ordenador para jugar a un videojuego o el propio conocimiento de cómo jugar.
} 


\subsection{Valoración de la experiencia}

Este factor integra 10 ítems que hacen referencia a las opiniones del alumnado respecto a la experiencia vivida y se alude a aspectos como el grado de atención que el videojuego requiere del usuario, diversión, planteamiento de estrategias para la superación de retos, cooperación como requisito para la misma, consideración como metodología de aprendizaje, así como la necesidad de jugar previamente al juego para facilitar la familiarización del usuario.

En los resultados obtenidos en esta dimensión, se registró que los estudiantes manifiestan una opinión de "parcialmente de acuerdo» en cuanto a la experiencia vivida en el uso del videojuego Portal 2 para la enseñanza-aprendizaje del francés como LE $(\mu=4.00 ; 6=.68)$.

Centrándonos en las diferencias que el sexo y la titulación establecían en función de la experiencia vivida en la dinámica de aprendizaje, la prueba $t$ de Student para muestras independientes señaló que no existían diferencias estadísticamente significativas en los dos casos.

Para conocer la influencia de la edad, el curso y los dispositivos con los que el alumnado juega en su vida cotidiana sobre los resultados se ha realizado un análisis de varianza a través de la prueba ANOVA de un factor. Los resultados obtenidos mostraron que tan solo existían diferencias estadísticamente significativas entre el curso y las opiniones de los estudiantes respecto a la experiencia vivida $[\mathrm{F}(2,105)=4.877$; $\mathrm{p}=.009]$. Las comparaciones múltiples post hoc, utilizando el estadístico de Tukey, permitieron conocer en qué cursos se encontraron dichas diferencias, siendo el alumnado de $4^{\circ}$ curso el que presentaba mayor valoración de la experiencia frente al alumnado perteneciente a $3^{\text {er }}$ y $2^{\circ}$ curso, respectivamente $(\mu=4.39$ vs $\mu=3.93$ y $\mu=3.87)$.

\subsection{Aportaciones del videojuego en la enseñanza-aprendizaje del francés}

Esta dimensión está formada por 11 ítems en los que se recogen las opiniones de los discentes en cuanto a las aportaciones del videojuego Portal 2 en la enseñanzaaprendizaje del francés, haciéndose referencia a elementos relacionados con la plataforma Steam, la mejora de la comprensión y expresión oral y escrita, así como el léxico y, también, al aprendizaje a nivel individual y grupal.

En los resultados obtenidos en este factor, se muestra que el alumnado manifiesta una valoración de indiferencia respecto a las aportaciones del videojuego Portal 2 en la enseñanza-aprendizaje del francés, $(\mu=3.51 ; 6=.80)$, aunque el valor está próximo a "parcialmente de acuerdo».

Por otra parte, la prueba $t$ de Student, realizada para identificar posibles diferencias con relación al sexo y la titulación del alumnado en cuanto a las aportaciones de la dinámica de enseńanza-aprendizaje del francés como LE mediante el videojuego Portal 2, indicó que no existían diferencias estadísticamente significativas en los dos casos. 
Asimismo, los análisis de varianza realizados para constatar la existencia de diferencias significativas entre la edad, el curso y los dispositivos para jugar del alumnado en relación con las aportaciones del videojuego Portal 2 en la enseñanza-aprendizaje del francés como LE mostraron que, al igual que en la dimensión anterior, únicamente existían diferencias estadísticamente significativas con relación al curso $[\mathrm{F}(2,105)=$ 5.962; $\mathrm{p}=.004]$. Las comparaciones múltiples post hoc, utilizando el estadístico de Tukey, mostraron en qué cursos se encontraron dichas diferencias, siendo de nuevo el alumnado de $4^{\circ}$ curso el que presentaba una mayor valoración en cuanto a las aportaciones de dicho videojuego en la enseñanza-aprendizaje del francés respecto a los que pertenecían a $2^{\circ}$ y $3^{\text {er }}$ curso, respectivamente $(\mu=4.03$ vs $\mu=3.40$ y $\mu=3.36)$.

\subsection{Calidad de sonidos/subtítulos}

Esta dimensión está compuesta por 4 ítems en los que se recoge las valoraciones de los estudiantes en cuanto a la calidad de los sonidos y subtítulos del videojuego Portal 2. Concretamente, se alude a las orientaciones de Wheatley (sistema), la ambientación sonora, la legibilidad de los subtítulos, así como la contribución de estos al propio juego. En los resultados obtenidos, se observa que los estudiantes manifiestan una valoración de indiferencia con relación a la dimensión «Calidad de los sonidos/subtítulos» $(\mu=3.37 ; 6=.85)$.

La prueba $t$ de Student, realizada para identificar posibles diferencias con relación al sexo y la titulación del alumnado en cuanto a la valoración de la calidad de los sonidos y subtítulos del videojuego, indicó que no existían diferencias estadísticamente significativas en ambos casos.

Por otra parte, los análisis de varianza realizados a través de la prueba ANOVA de un factor para constatar la existencia de diferencias significativas en función de la edad, el curso y los dispositivos empleados por el alumnado para jugar en cuanto a la valoración de la calidad de los sonidos y los subtítulos del videojuego, indicaron igualmente que no existían diferencias estadísticamente significativas en todos los casos.

\subsection{Recursos de apoyo usados durante la dinámica de aprendizaje}

La última dimensión del cuestionario está formada por 5 ítems en los que se alude a los recursos de apoyo usados por el alumnado durante la dinámica de aprendizaje, haciendo referencia al tutorial inicial del juego, así como a las consultas a los iguales, uso del diccionario, del traductor y ayuda del docente o alumnado colaborador.

En los resultados obtenidos, se muestra que los estudiantes manifiestan una valoración de indiferencia con relación a la dimensión «Recursos de apoyo usados durante la dinámica de aprendizaje» $(\mu=3.01 ; 6=.89)$.

La prueba $t$ de Student, realizada para identificar posibles diferencias con relación al sexo y la titulación del alumnado en cuanto a los recursos de apoyo empleados en la dinámica de aprendizaje, indicó que existían diferencias estadísticamente significativas en el primer caso $(\mathrm{T}=2.997, \mathrm{P}=.003)$. En este sentido, la media de los 
estudiantes del Grado de Maestro de Educación Primaria fue superior a los del Grado en Traducción e Interpretación (3.25 vs. 2.76).

Por otra parte, los análisis de varianza llevados a cabo a través de la prueba ANOVA de un factor para constatar la existencia de diferencias significativas en función de la edad, el curso y los dispositivos empleados por el alumnado para jugar, en cuanto a los recursos de apoyo usados durante la dinámica de aprendizaje, indicaron que únicamente existían diferencias estadísticamente significativas en el segundo caso $[\mathrm{F}(2,105)=9.254 ; \mathrm{p}=.000]$. Las comparaciones múltiples post hoc, utilizando el estadístico de Tukey, mostraron en qué cursos se encontraron dichas diferencias, siendo el alumnado de $3^{\circ}$ curso el que presentaba un mayor uso de los recursos de apoyo respecto a los que pertenecían a $4^{\circ}$ y $2^{\circ}$ curso, respectivamente, $(\mu=3.49$ vs $\mu=2.78$ y $\mu=2.76)$.

\subsection{Estudio correlacional}

En este apartado, se aborda el estudio correlacional entre los 4 factores del cuestionario, obtenidos en el AFE. Los datos resultantes de la aplicación de la prueba de correlación de Spearman pueden verse en la siguiente tabla (ver tabla 4).

\begin{tabular}{|c|c|c|c|c|c|c|}
\hline & & & Factor_1 & Factor_2 & Factor_3 & Factor_4 \\
\hline \multirow{12}{*}{$\begin{array}{c}\text { Rho de } \\
\text { Spearman }\end{array}$} & \multirow{3}{*}{ Factor_1 } & $\begin{array}{l}\text { Coeficiente } \\
\text { de correlación }\end{array}$ & 1.000 & $.778^{* *}$ & $.510^{* *}$ & .090 \\
\hline & & Sig. (bilateral) & & .000 & .000 & .354 \\
\hline & & $\mathrm{N}$ & 108 & 108 & 108 & 108 \\
\hline & \multirow{3}{*}{ Factor_2 } & $\begin{array}{l}\text { Coeficiente } \\
\text { de correlación }\end{array}$ & $.778^{* *}$ & 1.000 & $.634^{* *}$ & .118 \\
\hline & & Sig. (bilateral) & .000 & & .000 & .224 \\
\hline & & $\mathrm{N}$ & 108 & 108 & 108 & 108 \\
\hline & \multirow{3}{*}{ Factor_3 } & $\begin{array}{l}\text { Coeficiente } \\
\text { de correlación }\end{array}$ & $.510^{* *}$ & $.634^{* *}$ & 1.000 & .068 \\
\hline & & Sig. (bilateral) & .000 & .000 & & .482 \\
\hline & & $\mathrm{N}$ & 108 & 108 & 108 & 108 \\
\hline & \multirow{3}{*}{ Factor_4 } & $\begin{array}{l}\text { Coeficiente } \\
\text { de correlación }\end{array}$ & .090 & .118 & .068 & 1.000 \\
\hline & & Sig. (bilateral) & .354 & .224 & .482 & \\
\hline & & $\mathrm{N}$ & 108 & 108 & 108 & 108 \\
\hline
\end{tabular}

Tabla 4. Estudio correlacional de los 4 factores 
Los datos resultantes de dicha prueba confirman la existencia de relaciones entre el factor 1 (Valoración de la experiencia) con los factores 2 (Aportaciones del videojuego en la enseńanza-aprendizaje del francés) y 3 (Calidad de los sonidos/subtítulos), ya que $\mathrm{R}=.778 ; \mathrm{p}=.000$ y $\mathrm{R}=.510 ; \mathrm{p}=.000$, respectivamente, dado que hay nivel de significatividad bilateral al n.s=0.01. La relación entre las mismas es moderada y alta, respectivamente (Mateo, 2012).

Por otra parte, también se puede afirmar la existencia de relación entre el factor 2 (Aportaciones del videojuego en la enseñanza-aprendizaje del francés) con el factor 3 (Calidad de los sonidos/subtítulos), puesto que $\mathrm{R}=.634$; $\mathrm{p}=0.000$, y se da el nivel de significatividad bilateral al n.s=0.01. La relación entre las mismas es igualmente alta.

\subsection{Modelos explicativos de la valoración de la experiencia de aprendizaje}

En este punto se trata el factor «Valoración de la experiencia» en función de las medidas de los factores 2 (Aportaciones del videojuego en la enseñanza-aprendizaje del francés) y 3 (Calidad de los sonidos/subtítulos), a través de regresiones lineales múltiples (Pardo y Ruiz, 2002), con el fin de observar las variables predictoras y su relación con la variable criterio (ver tabla 5).

\begin{tabular}{|c|c|c|c|c|c|c|c|c|}
\hline \multicolumn{9}{|c|}{ Coeficientes $^{\mathrm{a}}$} \\
\hline & \multirow{2}{*}{ Modelo } & \multicolumn{2}{|c|}{$\begin{array}{l}\text { Coeficientes no } \\
\text { estandarizados }\end{array}$} & \multirow{2}{*}{$\begin{array}{c}\text { Coeficientes } \\
\text { estandarizados } \\
\text { Beta }\end{array}$} & \multirow[t]{2}{*}{$\mathrm{t}$} & \multirow[t]{2}{*}{ Sig. } & \multicolumn{2}{|c|}{$\begin{array}{l}\text { Estadísticas de } \\
\text { colinealidad }\end{array}$} \\
\hline & & B & $\begin{array}{l}\text { Error } \\
\text { estándar }\end{array}$ & & & & Tolerancia & VIF \\
\hline \multirow[t]{2}{*}{1} & $\begin{array}{l}\text { (Cons- } \\
\text { tante) }\end{array}$ & 1.670 & .190 & & 8.797 & .000 & & \\
\hline & Factor_2 & .662 & .053 & .773 & 12.556 & .000 & 1.000 & 1.000 \\
\hline
\end{tabular}

Tabla 5. Modelo predictor de la valoración de la experiencia

Los resultados obtenidos, tal y como se aprecia en la tabla 5, permiten observar que únicamente el factor 2 constituye un factor predictor del factor 1 , ya que $\beta=.773$, $t(773)=12.556, p<.01$. En este sentido, el valor de $R^{2}$ ajustado fue de .594 , indicando que un $59.4 \%$ de la variabilidad de la dimensión "Valoración de la experiencia» es explicada por el factor «Aportaciones del videojuego en la enseńanza-aprendizaje del francés».

\section{Discusión y conclusiones}

La incorporación de un videojuego cooperativo y su inclusión en sesiones didácticas ha supuesto un avance importante en la consideración del alumnado frente a otras consideraciones manifestadas sobre el uso de videojuegos serios (Brazo-Millán et al., 2018; Calvo-Ferrer, 2018; De Castro Castro et al., 2018). 
Tras analizar los resultados y el contexto en el que se desarrollaron nuestras sesiones, constatamos la existencia de dos situaciones de comunicación real en francés en el aula:

1. La establecida entre los alumnos en situación real de clase.

2. La de los jugadores, identificados con los avatares de los videojuegos.

En el primer contexto, que sigue siendo educativo, coincidimos con Barr (2017) y Muñoz González, Rubio García y Cruz Pichardo (2015) al comprobar que se puede establecer una situación de interacción real de aula y que dicha comunicación se potencia y acrecienta mediante el trabajo en línea, como también muestra el estudio de Chiong y Jovanovic (2012). La experiencia llevada a cabo incluía la comunicación en idioma extranjero como parte del reto al que sometíamos al alumnado. Consideramos que un buen videojuego debe ser difícil, de ahí su interés, de ahí también la satisfacción que produce el ir resolviéndolo. La inclusión de un medio de comunicación no habitual entre los jugadores no hace sino acrecentar el reto, pues es aceptado como otra orden o contrainte más del juego. La inmersión es total y sirve tanto a profesores como a los propios alumnos para valorar y evaluar su conocimiento real de lengua en interacción oral.

En el segundo contexto los resultados revelan que la inmersión en la tarea es total, cumpliendo a la perfección con la noción de tarea del enfoque accional y concordando, así, con los expuestos por Ollivier (2014) y Schmoll (2017). Los alumnos son realmente los avatares del juego (P-Body es el robot naranja, y Atlas, el azul). Coincidimos, por tanto, con otros estudios (Ebrahimzadeh y Alavi, 2017; Klimova y Kacet, 2017) en la selección de un videojuego comercial para desarrollar el proyecto, ya que Portal 2 ha sido muy galardonado entre usuarios y desarrolladores y no decepciona al alumnado universitario.

A diferencia del trabajo de Bado y Franklin (2014), nuestra experiencia de cooperación entre los alumnos no deja en ningún momento la comunicación en lengua extranjera y es capaz de crear una interacción cooperativa difícil de obtener en otro tipo de actividad.

Coincidimos en nuestros resultados, parcialmente, con el estudio de Klimova y Kacet (2017), pues el aprendizaje del vocabulario, no en forma reglada, sino como consecuencia de una comunicación sobre una tarea específica, ha sido percibido por los estudiantes como una necesidad para poderse comunicar entre las parejas y avanzar así en el juego. En nuestro caso, el videojuego propuesto y su contextualización propiciaban esta comunicación real. Por ello insistimos en el valor de la propuesta como medio de fomentar la interacción oral, actividad comunicativa que, como señala O’Dowd (2019), no suele formar parte de los trabajos de innovación educativa e investigación.

Podemos subrayar, por otra parte, que, aunque nuestra muestra no es muy numerosa, sí es representativa de la enseńanza universitaria de francés, en una universidad que tiene unos 400 alumnos de FLE, ya que esta lengua cuenta con muchos menos 
alumnos que el inglés como lengua extranjera. A este respecto, en primer lugar, se puede comprobar que el alumnado con mayor formación, de $4^{\circ}$ curso, que realiza la mención Francés, y por lo tanto más consciente de su aprendizaje, ha sabido valorar positivamente la inclusión de una sesión de videojuego en la que había que enfrentarse a retos lógico-espaciales, considerándola como una experiencia motivadora y novedosa: Me ha encantado. Al principio estaba un poco extrañada porque no creía que iba a ser tan entretenido. En mi opinión, creo que es bastante interesante, sobre todo, para utilizar esta metodología para enseñar y practicar la lengua.

La experiencia ha sido muy buena, ya que obligaba a hablar en francés y usar todas las técnicas posibles para que el compañero te entendiera.

En segundo lugar, hemos percibido una estrecha interacción entre alumnos colaboradores y alumnado de clase participantes de la experiencia, pues estos últimos han estado centrados en la solución de los retos planteados por el videojuego, lo que enriquece el valor cooperativo de la experiencia.

En tercer lugar, el alumnado ha destacado como principal aportación del videojuego para el aprendizaje de francés la práctica oral en una situación de comunicación real:

Aprendes a utilizar algunas expresiones en general, pero la tensión de haber estado en el juego te llevaba a que no pensaras si estabas expresándote correctamente.

Me he sentido satisfecha al comprobar que he sabido comunicarme en francés en un juego complicado como este.

Me ha parecido una experiencia gratificante para aprender léxico y comunicación ya que solo podíamos comunicarnos y pasar niveles a través de la comunicación oral y cooperativamente.

$\mathrm{Al}$ ser cooperativo he tenido que esforzarme mucho para comunicarle a mi compañera los problemas que había que resolver y lo que ella tenía que hacer.

Tener que hablar en francés me ha ayudado a expresarme mejor $\mathrm{y}$ he aprendido algunas palabras que he buscado.

El análisis de las grabaciones de los audios formará parte de nuestra próxima investigación y, aunque no hemos comenzado el análisis de los diálogos, por nuestra propia observación en el aula intuimos que abundarán las expresiones necesarias para establecer el contacto, función fática y emotiva de la lengua, así como aquellas que sirven para indicar qué se está haciendo o qué se debe hacer para conseguir avanzar en el juego, funciones conativa y referencial.

Desde un punto de vista de la organización de las sesiones, el poder jugar a un videojuego de manera cooperativa en una Facultad no técnica supone, en la actualidad, 
tener que solventar, a menudo de manera voluntariosa, unas carencias de infraestructuras presentes en la Universidad de Córdoba. Dichas carencias han implicado que alumnos y profesores deban traer su propio portátil, ratón, cascos con micrófono, teléfono móvil con aplicación de grabación de audio, incluso conexiones eléctricas, y que estén sometidos a una WIFI débil e inconstante. Todo ello reduce la comodidad y disposición del alumnado a participar en estas sesiones.

Aunque el nivel medio de FLE de los alumnos podría considerarse que es un B1, algunos de ellos tienen un nivel superior (B2). No obstante, también existe alrededor de un 20\% de estudiantes que tienen un nivel más bajo al B1 y que manifiesta su dificultad de comunicarse en francés de una forma fluida:

No me expreso con claridad en francés.

La mayor dificultad ha sido la comunicación con el compañero, porque no manejo el vocabulario.

La principal dificultad era entender hablar francés a mi compañera $y$, también, los diferentes niveles de juego en los que no sabíamos lo que teníamos que hacer.

La mayor dificultad era hacerme entender con mi compañera en francés.

La mayor dificultad: expresarme bien en francés, aunque a medida que he avanzado en el juego, he empezado a notar que cada vez hablaba con más fluidez.

La mayor dificultad era a la hora de comunicarme en francés con mi compañera, pero con la ayuda del traductor ha sido más fácil.

Dada la posibilidad de utilizar recursos en línea, como diccionario o traductor, los alumnos con menor dominio de la lengua francesa y dificultades de comprensión lingüística recurrieron a ellos, aunque no fue una práctica habitual, ya que tanto la cooperación creada en el aula entre ellos como la ayuda de los profesores y colaboradores permitían aclarar sus dudas. A este respecto, alumnos con buen dominio del francés y con buen dominio del juego llegaron a niveles superiores o lo finalizaron completamente frente a aquellos cuyo nivel lingüístico y de juego era menor y necesitaban todo tipo de ayuda, quedándose en los primeros niveles.

Apostar por el uso del videojuego en la enseñanza de idiomas y que este se convierta en una práctica habitual, cómoda y enriquecedora, es necesario. Así lo manifestaban nuestros estudiantes mientras jugaban en el aula, pues, a pesar de las dificultades crecientes en la superación de los niveles, el grado de comunicación con el compańero de juego aumentaba a medida que se avanzaba, resultando para ellos en una cooperación más fácil:

He llegado al nivel $7 \mathrm{y}$ algunos niveles me han parecido bastante complicados, pero conforme se avanza, se aprende más y lo que antes era difícil en el siguiente nivel era fácil y obvio. 


\section{REFERENCIAS BIBLIOGRÁFICAS}

BADO, Niamboue \& Teresa FranKLIN (2014): «Cooperative Game-based Learning in the English as a Foreign Language Classroom». Issues and Trends in Educational Technol$o g y, 2:$ 2. DOI: https://doi.org/10.2458/azu_itet_v2i2_bado

BARR, Matthew (2017): «Video games can develop graduate skills in higher education students: A randomised trial». Computers \& Education, 113, 86-97. DOI: https://doi.org/10.1016/j.compedu.2017.05.016

BRAZO-MILLÁN, Ana Isabel (2020): «El videojuego en la enseñanza de FLE: se puede aprender francés mediante videojuegos», in Natividad Aguayo Arrabal (ed.), Educación de las Segundas Lenguas. Córdoba, UCOPress, 69-73.

BraZO-Millán, Ana Isabel; Juan Manuel MuÑOZ GONZÁleZ \& Carmen CASTRO DE CASTRO (2018): «Aprendiendo léxico y ortografía francesa en la universidad mediante el videojuego Scribblenauts». EDMETIC, Revista de Educación Mediática y TIC, 7: 2, 1836. DOI: https://doi.org/10.21071/edmetic.v7i2.7201

CASAÑ-PITARCH, Ricardo (2017): «Storyline-Based Videogames in the FL Classroom». Digital education, 37, 80-92.

CHIONG, Raymond \& Jelena JOVANOVIC (2012): «Collaborative learning in online study groups: An evolutionary». Journal of Information Technology Education: Research, 11: 1, 81-101. URL: https://www.learntechlib.org/p/111494

CONSEJO De EUROPA (2002). Marco Común Europeo de Referencia para las Lenguas: aprendizaje, enseñanza, evaluación. Madrid, Ministerio de Educación, Cultura y Deporte.

CREIGHTON, Susan \& Andrea SZYMKOWIAK (2014): «The Effects of Cooperative and Competitive Games on Classroom Interaction Frequencies». Procedia - Social and Behavioral Sciences, 140, 155-163.

DAYOUB, Dima (2019): «Vernacular Digital Games as English Language Learning Tools: An Exploratory Study in the Syrian Context». Tishreen University Journal for Research and Scientific Studies - Arts and Humanities Series, 41: 2. 791-804.

De Castro Castro, Carmen; Juan Manuel MuÑoz GonZÁleZ, \& Ana Isabel BraZO-MILLÁN (2018): «El uso de videojuegos serios en el aprendizaje de francés en educación superior». Revista Mexicana de Investigación Educativa, 23: 76. 157-177.

DE HAAN, Jonathan (2011): «Teaching and learning English through digital game projects». Digital Culture \& Education, 3: 1, 46-55.

DICKEY, Michelle (2011): «Murder on Grimm Isle: The impact of game narrative design in an educational gamebased learning environment». British Journal of Educational Technology, 42: 3, 456-469.

EBRAHIMZADEH, Mohsen \& Sepideh ALAVI (2017): «The effect of digital video games on EFL students' language learning motivation». Teaching English with Technology, 17: 2, $87-112$.

HIGGINS, Steven; Zhimin XIAO \& Maria KATSIPATAKI (2012): The impact of digital technology on learning: A summary for the education endowment foundation. Full Report. Durham, Durham University. 
KeSSLER, Carolyn [ed.]. (1992): Cooperative Language Learning: A Teacher's Resource Book. Englewood Cliffs, NJ, Prentice Hall Regents.

KINZIE, Mable \& Dolly JOSEPH (2008): «Gender differences in game activity preferences of middle school children: implications for educational game design». Educational Technology Research and Development, 56: 5-6, 643-663. DOI: https://doi.org/10.1007/s11423-007-9076-z

KLIMOVA, Blanka \& Jaroslav KACET (2017): «Efficacy of Computer Games on Language Learning». Turkish Online Journal of Educational Technology - TOJET, 16: 4, 19-26.

LorenZO-SEVA, Urbano (2000): «The weighted oblimin rotation». Psychometrika, 65, 301318.

MATEO, Juan (2012): «La investigación expost-facto», in Rafael Bisquerra (coord.). Metodología de investigación educativa, Madrid, La Muralla, 195-229.

MERINO-SOTO, César (2016): «Diferencias entre coeficientes alfa de Cronbach, con muestras y partes pequeñas: Un programa VB». Anales de Psicología, 32: 2, 587-588.

MuÑoz GONZÁleZ, Juan Manuel; Sebastián Rubio GARCía \& Ivanovnna M. CRUZ PICHARDO (2015): «Strategies of collaborative work in the classroom through the design of video games». Digital Education Review, 27, 69-84.

O'DOWD, Robert (2019): «Evaluating the impact of telecollaborative exchange in university education». Alsic, 22: 1. DOI: https://doi.org/10.14705/rpnet.2018.jve.1

OlLIVIER, Christian (2014): «Vers une approche interactionnelle en didactique des langues et une extension du domaine de la tâche - Les atouts du web 2.0». Alsic, 17. DOI: https://doi.org/10.4000/alsic. 2743

OTZEN, Tamara \& Carlos MANTEROLA (2017): «Técnicas de muestreo sobre una población a estudio». International Journal of Morphology, 35: 1, 227-232. DOI: http://dx.doi.org/10.4067/S0717-95022017000100037

PARDO, Antonio \& Miguel Ángel RUíZ (2002). SPSS 11: Guía para el análisis de datos. Madrid, Mcgraw-Hill.

Rosas, Ricardo, Miguel Nussbaum, Patricio Cumsille, Vladimir Marianov, Mónica CoRREA, Patricia FlORES, ... \& Marcela SALINAS (2003): «Beyond Nintendo: Design and assessment of educational video games for first and second grade students». Computers \& Education, 40: 1, 71-94.

SCHMOLL, Laurence (2017): «Penser l'intégration du jeu vidéo en classe de langue». Recherche et pratiques pédagogiques en langues de spécialité, 36:2. DOI: https://doi.org/10.4000/apliut.5722

THORNE, Steven, Rebecca BLACK \& Julie SYKES (2009): «Second language use, socialization, and learning in Internet interest communities and online gaming». The Modern Language Journal, 93, 802-821.

TIMMERMAN, Marieke E. \& Urbano LORENZO-SEVA (2011): «Dimensionality Assessment of Ordered Polytomous Items with Parallel Analysis». Psychological Methods, 16: 2, 209220. 


\section{ANEXO}

Preguntas abiertas del cuestionario denominado Cuestionario sobre la enseñanza-aprendizaje del francés a través del videojuego Portal 2.

1. Las principales dificultades que he tenido han sido...

2. He llegado al nivel... y me ha parecido...

3. Coméntanos cómo has vivido la experiencia de aprendizaje del francés a través de un videojuego cooperativo. 